Proceeding

\section{Prevent BP/diabetes}

Keywords: diabetes, sugar, fructose, insulin, hypertension

\section{Can we prevent BP and diabetes naturally?}

Yes. We have helped Thousands of our clients to prevent \& reverse their BP \& Diabetes Naturally within 4-6 months. Hypertension is typically a symptom of insulin and leptin resistance. And the vast majority of those who have hypertension can normalize their blood pressure without resorting to drugs. The best natural solution to hypertension and diabetes is to monitor your diet and lifestyle by following the wellness IQ at this website.

We have helped thousands of our clients worldwide to normalize both hypertension and diabetes within 3 months and they accordingly reduced and eventually cut their medications within 4-6 months.

One of the primary underlying causes of high blood pressure is related to your body producing too much insulin and leptin in response to a high-carbohydrate and processed food diet. Avoid processed foods, as most are loaded with sugar/fructose, processed salt, and harmful fats like trans fat and damaged omega-6-rich vegetable oils. Instead, focus on intermittent fasting to boost results which will help normalize your insulin/leptin sensitivity. This is crucial for normalizing your blood pressure. Also consider whole food, ideally organic diet. Meats should ideally be grass fed and pasture finished. Also keep an eye on your sodium to potassium and omega-3/omega- 6 fat ratios. This is more or less automatically done if you swap processed foods for whole foods.

As your insulin and leptin levels rise, it causes your blood pressure to increase. Research published in 1998 in the journal Diabetes reported that nearly two-thirds of the test subjects who were insulin resistant also had high blood pressure, so the link is significant.

Elevated uric acid levels are also significantly associated with hypertension, so any program adapted to address high blood pressure needs to help normalize both your insulin sensitivity and uric acid level.

As it turns out, by eliminating excess sugar/fructose from your diet, you can address all three issues in one fell swoop. Other treatment strategies that I will discuss below also tend to have this effect.

\section{Blood pressure classification systolic pressure $(\mathrm{mmHg})$ diastolic pressure $(\mathrm{mmHg})$}

Normal Pre-hypertension 120-139/ 80-89

Stage 1Hypertension 140-159/ 90-99

Stage 2 Hypertension 160/100+

Hypertension is also categorized as either primary or secondary hypertension. The former applies to about 90-95 percent of those with high blood pressure, and while the conventional medical establishment claim the cause is idiopathic or unknown, primary hypertension (aka essential hypertension) is more than likely linked to insulin/leptin resistance.
Volume 2 Issue 2 - 2018

\section{George Grant}

World organization of Natural Medicine, CEO of Academy of Wellness, Canada

Correspondence: George Grant, World organization of Natural Medicine, CEO of Academy of Wellness, 17 Waterhouse Way, Richmond Hill, ON L4C 9H8, Ontario, Canada, Tel + I 416562-3140,Email drgrant@rogers.com

Received: January 24, 2018 | Published: March 29, 2018

Secondary hypertension applies to the remaining five to I 0 percent whose high blood pressure is caused by chronic liver disease

In addition to checking your blood pressure, I highly recommend getting a fasting insulin level test done. Remember, high blood pressure and insulin resistance tend to go hand-in-hand, and if your hypertension is the result of elevated insulin levels, you will know how to nip it in the bud by the end of this article. Prevention is always better than cure. Meditation is better than medication for both hypertension and diabetes.

I recommend the following natural herbal remedies to moderate hypertension \& diabetes:

a) Turmeric: $100 \%$ Effective in Preventing Diabetes and hypertension.

b) Ginger: Lowers Fasting Blood Glucose by $10.5 \%$ as well as hypertension.

c) Cinnamon: Less Than Half a Teaspoon a Day Reduces Blood Sugar Levels and high blood pressure.

d) Olive Leaf Extract: Results Comparable to Metformin and drugs for hypertension

e) Berries Lower After-Meal Insulin Spike and lowers blood pressure.

f) Black Seed (Nigella Sativa): Just 2 Grams Reduces Insulin Resistance and hypertension.

g) Spirulina helps with insulin resistance and hypertension. See the complete blog under Spirulina.

h) Berberine Just as Good as Three Different Diabetes Drugs and high blood pressure medications. 
A very great king wrote the following prayer to God, You made all the delicate, inner parts of my body and knit me together in my mother's womb. Thank you for making me so wonderfully complex! Your workmanship is marvelous how well I know it. His words ring so true when it comes to potassium and its role in the human body.

\section{Potassium is the natural solution to hypertension \& diabetes}

You and I cannot live without potassium. Potassium is chief among those minerals called electrolytes. Other electrolyte minerals include: sodium, chloride, calcium, magnesium and phosphorus.

\section{Energy and potassium}

Potassium is the most commonly measured mineral in the blood. We each carry around a little over a quarter pound (120 grams) of this precious mineral, but 98 percent of it is distributed in minuscule portions throughout the cells of our bodies.

In our cells, potassium serves as the primary positive ion responsible for a myriad of biochemical and electrical functions. Potassium plays a significant role in:
i. Regulating the heartbeat
ii. Building and operation of the muscles
iii. Maintaining bone health
iv. Keeping blood pressure down
v. Assisting the cells in the production of proteins
vi. Sending electrical impulses to the nerves
vii. Maintaining normal body growth
viii. Metabolizing carbohydrates
ix. Sustaining energy levels

\section{Where does potassium come from?}

The body cannot produce potassium, but most of us get all the potassium we need from eating healthy foods. In fact, nearly all natural foods (from living organisms) contain potassium.

However, it is possible to suffer from hyperkalemia (too much potassium) or hypokalemia (too little potassium). Hyperkalemia usually only occurs when there are other serious medical issues. Under normal circumstances, the kidneys function to rid the body of too much potassium.

\section{Hypokalemia is more common and can be brought on by many factors including:}

a. Diarrhea

Vomiting

Other gastrointestinal problems

b. Excessive sweating

c. Diuretics

Eating a high sodium diet d. Poor diet of too much sugar and junk food

\section{Severe hypokalemia can lead to very serious consequences and even death. Symptoms of hypokalemia can include:}

a) Fatigue

Weakness in the muscles

b) Slow reflexes

c) Dry skin or acne

d) Cardiac arrhythmia

e) Muscle cramps

f) Impairment of glucose metabolism

g) Insomnia

Anorexia

Depression

Nervous disorders

If you engage in sports or other physical activity at a higher than moderate level, you may experience the fatigue, muscle cramps and other symptoms associated with loss of electrolytes. When you exercise hard, you lose these minerals through your sweat.

There is an epidemic of blood sugar problems in America and scientists now believe they have found out the reason why.

Scientists tested 2,016 Americans for the presence of six toxins known as POPs (persistent organic pollutants). The scientists then compared the levels of these 6 toxins in the participant's bodies to their history of blood sugar problems.

And here is what they discovered. The people with the highest levels of these 6 toxins were 38 times more likely to have blood sugar problems compared to the people with the lowest levels!

That is because these toxins can destroy the beta cells in your pancreas that produce insulin, the hormone that regulates your blood sugar levels. These toxins can also interfere with the cell receptors that carry glucose (sugar) to your muscle and fat cells. And they can limit your cell receptors ability to use insulin. When your cells have a hard time accepting and using insulin, then your blood sugar rises.

In the October 2014 IHN a novel dietary approach was discussed which successfully reversed type 2 diabetes in 8 weeks. The research group headed by Dr. Roy Taylor of the University of Newcastle in the UK has just published a second clinical trial.17 This trial addresses the question of the efficacy of the diet for individuals with long-term duration of diabetes ( $>8$ years) as compared to those with short-term duration $<4$ years), the latter group being similar to those the original study published in 2011.18 The report was prefaced by the statement: The inevitably progressive nature of type 2 diabetes has been widely accepted since the UK Prospective Diabetes Study was carried out, which showed that glucose control steadily worsened towards requirement for insulin treatment despite best possible therapy. There were 15 subjects in the short-duration and 14 in the long-duration groups. The protocol was similar to the earlier study with subjects taken off anti-diabetic medications prior to the start and then all put on 
a diet of approximately 700 calories for 8 weeks. The diet consisted of 600 calories from a meal replacement product and the balance from vegetables. Using fasting blood glucose (FBG) as the criterion, all the subjects in the short-duration group regressed to normal glycemia and could be considered at least temporarily cured.

\section{Hypertension: controlling the 'silent killer'}

This report lays out a step-by-step lifestyle program you can use to lower your blood pressure, including a special section on the best diet for keeping your blood pressure in check. It also covers blood pressure monitoring and medications. With the information available today, there is no need for hypertension to be a killer any longer.

The easiest way to lower blood pressure is by popping a pill. It takes just a few seconds and you are finished. But pills cost money and often have side effects. Fully relaxing your body and your mind for a few minutes a day could lower your systolic blood pressure (the top number of a blood pressure reading) by 10 points or more at no cost, and with no side effects.

Researchers with the Benson-Henry Institute for Mind-Body Medicine at Harvard-affiliated Massachusetts General Hospital compared a stress management technique called the relaxation response with education about lifestyle changes such as sodium reduction, weight loss, and exercise. They found:

Blood pressure decreased more in the relaxation response group $32 \%$ of the volunteers in that group were able to eliminate one blood pressure medication and still keep their blood pressure under control, compared with $14 \%$ of the lifestyle group.

The relaxation response, developed by Harvard's Dr. Herbert Benson, has been shown to reduce heart rate, blood pressure, breathing rate, and muscle tension. Here's how to do it:

a. Select a word (such as "one" or "peace"), a short phrase, or a prayer to focus on.

b. Sit quietly in a comfortable position and close your eyes.

c. Relax your muscles, progressing from your feet to your calves, thighs, abdomen, and so on, up to your neck and

d. face.

Breathe slowly through your nose, silently saying your focus word, phrase, or prayer to yourself as you exhale.

e. When other thoughts come to mind, don't worry. Simply return your attention to your focus word, phrase, or prayer.

f. Do this for 10 to 20 minutes.

g. Sit quietly for a minute or so, and then open your eyes.

h. Practice the relaxation response once or twice a day.

\section{Blood pressure basics}

Blood pressure is the force that a wave of blood propelled from the heart exerts on the arteries. It is measured at two points; each measurement is recorded in millimeters of mercury $(\mathrm{mm} \mathrm{Hg})$.

Systolic pressure gauges the pressure in the arteries at systole (SIS-tuh-lee), the instant when the heart contracts and pushes a wave of blood along the arterial tree (think "s" for squeeze). It is the top number of a blood pressure reading.

Diastolic pressure reflects the pressure during diastole (die-AStuh-lee), the brief period of relaxation between beats. It is the bottom number of a blood pressure reading.

\section{Hypertension is the formal name for high blood pressure}

Blood pressure categories

Systolic Diastolic

Normal (optimal) less than 120 and less than 80

Prehypertension $120-139$ or $80-89$

Hypertension 140 or higher or 90 or higher

Many studies support the belief that having an upbeat and positive attitude will translate into living a longer, healthier life, and conversely, that a pessimistic outlook promotes ill health and can shave years off your life.

For example, in one study, 1 the tendency to always expect the worst was linked to a 25 percent higher risk of dying before the age of 65 .

Perhaps one of the most well-known forerunners of "the science of happiness" was Norman Cousins, who in 1964 was diagnosed with a life-threatening autoimmune disease.

After being given a 1 in 500 chance of recovery, Cousins created his own laughter therapy program, which he claims was the key to his ultimate recovery. He went on to write the book, "Anatomy of an Illness," and established the Cousins Center for Psychoneuroimmunology2 in Los Angeles, California. Without a correct breathing you have a poor cells oxygenation, an acidic $\mathrm{pH}$ of the blood, a low mitochondrial energy, a perfect terrain for cancer and all kind of degenerative diseases. If your breathing stops you are dead in max. $3 \mathrm{~min}$, isn't this a primordial condition to look after? The first thing we do when born is breathing and interestingly enough it is also what deteriorates at the fastest path during our life; along with worsening the breath habits, we must question if this is not the real reason of aging! Aging is actually a deterioration of our respiration with metabolic consequences and organs degeneration due to lack of oxygen, an acidification of cells, calling for a progressing cells apoptosis.

\section{Deep breathing can lower your BP by I0\% without medications}

Modern fitness research offers many potent reminders that physical activity is one of the best "preventive drugs" for many common ailments, from psychiatric disorders to heart disease, diabetes and cancer.

Many studies have also confirmed that prolonged sitting is an independent risk factor for disease and early death. So it's no major surprise to find that inactivity may be costing the global economy tens of billions of dollars each year in lost productivity and healthcare costs. 
As reported by Reuters and others, a recent study looking at data from 1 million individuals worldwide found that lack of physical activity had a global price tag of $\$ 67.5$ billion in 2013 .

Of this amount, $\$ 32.2$ billion was paid by the public sector, $\$ 12.9$ billion by the private sector and $\$ 9.7$ billion by individual households. According to their findings, one hour of daily exercise could eliminate a majority of these expenses.

According to the researchers, inactivity is also the cause of more than 5 million deaths per year. To put that into perspective in terms of being a risk factor, smoking kills about 6 million annually.

Studies dating back nearly a century noted a striking finding: If you take young, healthy people and split them up into two groups half on a fat-rich diet and half on a carbohydrate-rich diet we find that within just two days, glucose intolerance skyrockets in the fat group. The group that had been shoveling fat in ended up with twice the blood sugar. As the amount of fat in the diet goes up, so does ones blood sugar. Why would eating fat lead to higher blood sugar levels? It would take scientists nearly seven decades to unravel this mystery, but it would end up holding the key to our current understanding of the cause of type 2 diabetes.

The reason athlete's carb-load before a race is to build up the fuel supply within their muscles. We break down the starch into glucose in our digestive tract, it circulates as blood glucose (blood sugar) and is taken up by our muscles to be stored and burnt for energy.

Blood sugar, though, is like a vampire. It needs an invitation to come into our cells. That invitation is insulin. Insulin is the key that unlocks the door that lets glucose in the blood enter muscle cells. When insulin attaches to the insulin receptor on the cell, it activates an enzyme, which activates another enzyme, which activates two more enzymes, which finally activates glucose transport (as diagrammed in my video What Causes Insulin Resistance?).

What if there was no insulin? Blood sugar would be stuck in the bloodstream banging on the door to our muscles, unable to get inside. With nowhere to go, sugar levels in the blood would rise and rise. That's what happens in type 1 diabetes: the cells in the pancreas that make insulin get destroyed, and without insulin, sugar in the blood can't get out of the blood into the muscles, and so blood sugar rises. But there is a second way we could end up with high blood sugar.

What if there enough insulin, but the insulin doesn't work? The key is there, but something's gummed up the lock. This is insulin resistance. Our muscle cells become resistant to the effect of insulin. What's gumming up the locks on our muscle cells? What's preventing insulin from letting glucose in? Tiny droplets of fat inside our muscle cells, so-called intramyocellular lipid.

Fat in the bloodstream can build up inside the muscle cell, creating toxic fatty breakdown products and free radicals that block the insulin signaling process. No matter how much insulin we have in our blood, it's not able to sufficiently open the glucose gates and blood sugar levels build up in the blood. And this can happen within three hours. One hit of fat can start causing insulin resistance, inhibiting blood sugar uptake after just 160 minutes.

This mechanism by which fat induces insulin resistance wasn't known until fancy MRI techniques were developed to see what was happening inside people's muscles as fat was infused into their bloodstream. That's how we found that elevation of fat levels in the blood causes insulin resistance by inhibition of glucose transport into the muscles.

We can also do the opposite experiment. Lower the level of fat in people's blood and the insulin resistance comes right down. If we clear the fat out of the blood, we also clear the sugar out. That explains the finding that on the high fat, ketogenic diet and insulin doesn't work very well. Our bodies become insulin resistant. But as the amount of fat in our diet gets lower and lower, insulin works better and better a clear demonstration that the sugar tolerance of even healthy individuals can be impaired by administering a lowcarb, high-fat diet. We can decrease insulin resistance, however, by decreasing fat intake.

The effect is really dramatic check out at least the end of my video What Causes Insulin Resistance? to see what happens as dietary fat intake drops.

The most concerning downside of low-carb diets, though, is heart health: Low Carb Diets and Coronary Blood Flow.

\section{Acknowledgment}

Author declares no acknowledgment.

\section{Conflict of interest}

Author declares no conflict of interest. 